\section{Pressotherapy with adjustable high-pressure gradients}

\section{Claude-Julien Cartier \\ Cabinet Médical, Châtearoux, France}

\begin{abstract}
Our team is presenting a new therapeutic concept based on the important experience acquired around the mercury fluid's strong internal hydrostatic pressures in vascular and post-traumatic therapy. Currently, the objective is to extend the physical recourse to strong pressures without mercury to carry on the advantages and open up to other performances.
\end{abstract}

\section{Context}

When looking for the tissue complications of scuba diving in the literature, we do not find any. Yet the soft parts of the body receive 1 bar of extra pressure every 10 meters deep. In apnea, we can easily reach 3 to 10 extra bar. With bottles, we go down much deeper and a lot longer.

1 bar is the atmospheric pressure on the cutaneous surfaces at sea level. 1 extra bar is a familiar pressure for the plantar lymphatics when walking, and this pressure is greatly increased during jogging and jumping. 1 bar is the maximum pressure of the high gradients known and used on the human body (mercury).

Professor Henri Boccalon (Vascular Physician, Rangueil University Hospital of Toulouse), performed isotopic lymphoscintigraphy in 1992 before and after treatment with Mercurial Pressotherapy of 30 primary and secondary lymphoedemas of the lower limbs by monotherapy: i) 13 flow improvements; ii) 16 unchanged states; iii) 1 single aggravation on recurrence of cancer, with clinical improvement.

Today, after 30 years of Mercurial Pressotherapy and more than 2,000,000 patients treated (medical practices, hospitals): no early or late deleterious effects in the usual results on loosening, volume reduction, healing. ${ }^{1-5}$

Therefore, all these data seem to allow us to go further in the use of strong pressures safely applied to vascular diseases in limbs.

\section{Argumentary}

How and why apply high-pressure gradients?

We know the physiological interest of the continuous diurnal compression stokings on liquid transfers (10 to $40 \mathrm{mmHg}$ ).

The compression can also be applied in a discontinuous, and even paroxysmal mode: i) equal pressure: nothing happens and the tissue fluids settle in depth; ii) degressive compression with low pressure gradient (=existing pressotherapies, less than $200 \mathrm{mmHg}$ ): the action is absorbed quickly in the first support tissues, in proportion to the magnitude of the applied pressure gradient; iii) this explains the major physiological interest of applaing high pressure gradients, between 200 $\mathrm{mmHg}$ and $760 \mathrm{mmHg}$ (level of the mercury pressotherapy).

In the compressed territories, volume decrease and the relaxation of the interstitium and lymphatic canals restores the necessary flexibility to the physiological action of pumping macro-proteins; as soon as the pressures get stronger, the action is transmitted in depth, where the tissues are the most diversified and the lymphatic vessels are the rarest, physiologically overflowing: all the support tissues, muscular compartments, aponeuroses, neurovascular sheaths, tendons, articular areas, periosteum; this action involves moreover a tightening effect on the arteriolar vasodilator endothelium with reactive hyperaemia and macroscopic permanent erythrosis. Here, the vasodilatation takes place in a territory emptied of any venous content to stimulate the increase of the tissue oxygenation turn over. This has been schown by the study carried out at INSEP by Dr. Jousselin in 1990, which demonstrates, on the lactate levels decreases, the acceleration of the return to muscular aerobiosis in active sports recovery with strong pressure gradient, which was not atchieved with the low-pressure therapies that have been tested.

\section{Method}

The treatment that we propose is a gradual paroxysmal discontinuous compression.

- Initial treatment: 3 to 4 sessions of 30 minutes spaced at intervals of 1 to 8 days.

- Maintenance treatment: 1 session after 1 month, then 3 months, then every 6 months or once a year and when necessary according to evolution. The patient is lying down and relaxed while leaning his torso.
Correspondence: Claude-Julien Cartier, Cabinet Médical, 7 Rue Albert 1er, 36000 Châteauroux, France.

E-mail: mlaure.cartier11@yahoo.fr

Conference presentation: International Compression Club (ICC) Meeting, Paris, 2017

This work is licensed under a Creative Commons Attribution 4.0 License (by-nc 4.0).

CC Copyright C-J. Cartier, 2018

Licensee PAGEPress, Italy

Veins and Lymphatics 2018; 7:7622

doi:10.4081/vl.2018.7622

\section{Equipment}

We turn to compressed air in a characteristic stack of multiple pockets, using a gradient smoothing method on the limbs.

Variable pressure gradients ranging from the upper limit of the existing pressotherapy to the studied hydrostatic pressures of mercury are programmed.

The maximum peripheral pressures can be reported higher on the limb. In pure lymphatic pathologies, the device dispenses proximal ganglionic pressures. The contention is widely used around treatments.

\section{Input}

What is innovative and makes a difference with the existing Pressotherapies:

- $\quad$ the set of pressure macro gradients $(0,2$ to 1 bar);

- the deep action;

- the transfer of the maximum pressures;

- the evacuation/vasodilatation conjunction;

- the induced aerobiosis.

Compared with Mercurial Pressotherapy:

- the performance without mercury;

- the absence of environmental constraint;

- the position at rest during treatment;

- the small size of the device, its mobility, its lightness, its cost.

Proposed pressure gradients will have 3 possible uses:

- medical and paramedical (the first to be developed);

- $\quad$ sports fields, fitness, spas;

- well-being and aesthetics.

\section{Target of the medical environ- ment}

- Venous and veno-lymphatic pathologies 
(sequelae of thrombosis, pain, heaviness, edemas, hypodermitis, ulcers);

- Sequelae of trauma (sprains, fractures, tears, algoneurodystrophy);

- Sport pathologies and traumas;

- Primary and secondary lymphedemas, early or old;

- Prevention of late edemas;

- Mixed pathologies;

- Contraindicated arterial surgeries for the elderly.
Currently, the proof of concept is established and we will produce the prototypes in spring, for technical and then medical experiments.

\section{References}

1. Cartier CJ. Mercury bath pressotherapy, indications, results. Phlebologie-SFP
1988.

2. Cartier CJ. Pressoyherapy by mercury in the treatment of limphedemas. J Malad Vascul 1990;15.

3. Cartier CJ. Edema physical treatment by mercury. Eur J Lymphol 1992;3:10.

4. Cartier CJ. Mercury pressotherapy (P.M.). Angiologie 2000;52.

5. Schadeck M. Pressotherapy with bath of mercury in the hypodermitis treatment. Phlebologie-SFP 2001;54. 